Pacific Journal of Mathematics

ON THE GROWTH OF MEROMORPHIC FUNCTIONS WITH
RADIALLY DISTRIBUTED ZEROS AND POLES 


\title{
ON THE GROWTH OF MEROMORPHIC FUNCTIONS WITH RADIALLY DISTRIBUTED ZEROS AND POLES
}

\author{
JOSEPH MiLeS
}

The lowest possible rate of growth of a meromorphic function $f$ of genus $q$ with zeros and poles restricted to a given finite set of rays through the origin is determined in terms of $q$ and the rays carrying the zeros and poles. For $\alpha>1$ the ratio $T(\alpha r, f) / T(r, f)$ is shown to be bounded as $r$ tends to infinity for all such entire functions, but not for all such meromorphic functions.

1. Introduction. In this paper we are concerned with the rate of growth of the Nevanlinna characteristic of meromorphic functions whose zeros and poles are restricted to lie on a finite number of rays through the origin. We consider the relationship between the order and lower order of such functions as well as upper bounds for $T(\alpha r, f) / T(r, f)$ for $\alpha>1$.

We first specify the class of functions that we will consider. Suppose $X=\left\{\theta_{1}, \theta_{2}, \ldots \theta_{M}\right\}$ and $\left\{Y=\theta_{M+1}, \theta_{M+2}, \ldots \theta_{L}\right\}$ each consist of distinct members of $[0,2 \pi)$, are not both empty, and have an empty intersection. For a nonnegative integer $q$, let $\mathscr{M}_{q}(X, Y)$ be the collection of all functions meromorphic in the complex plane with zeros $z_{\nu}$ and poles $w_{\nu}$ satisfying

$$
\begin{aligned}
& \arg z_{\nu} \in X, \\
& \arg w_{\nu} \in Y, \\
& \sum_{\nu} \frac{1}{\left|z_{\nu}\right|^{q}}+\sum_{\nu} \frac{1}{\left|w_{\nu}\right|^{q}}=\infty,
\end{aligned}
$$

and

$$
\sum_{\nu} \frac{1}{\left|z_{\nu}\right|^{q+1}}+\sum_{\nu} \frac{1}{\left|w_{\nu}\right|^{q+1}}<\infty
$$

For $X \neq \varnothing$, let $\mathscr{E}_{q}(X)$ be the collection of entire functions $\mathscr{M}_{q}(X, \varnothing)$. We note it is immediate from (1.1iii) that $f \in \mathscr{M}_{q}(X, Y)$ has order $\lambda \geq q$.

Our principal result (Theorem 1) enables us to determine the minimum of the lower orders $\mu$ of $f \in \mathscr{M}_{q}(X, Y)$ by applying a certain criterion, essentially geometric in character, to the sets

$$
S_{k}=\left\{e^{-i k \theta_{j}}: 1 \leq j \leq M\right\} \cup\left\{-e^{-i k \theta_{J}}: M+1 \leq j \leq L\right\}
$$


for $0 \leq k \leq q$. Theorem 1 extends earlier results of Edrei and Fuchs [1, p. 308], Gol'dberg [5] and [6, pp. 338-344], and Steinmetz [11], who obtained the sharp bounds $\mu \geq q$ for $f \in \mathscr{E}_{q}(X)$ if $M=1$ ([1] and [5]) and $\mu \geq \max (0, q-1)$ for $f \in \mathscr{E}_{q}(X)$ if $M=2$ ([5] and [11]).

THEOREM 1. Let the nonnegative integer $p=p(q, X, Y)$ be associated with the class $\mathscr{M}_{q}(X, Y)$ in the following way.

(a) If $q=0, p=0$.

(b) Suppose $q \geq 1$. For each integer $m_{0}, 0 \leq m_{0} \leq q$, consider the system of $q-m_{0}+1$ equations

$$
\sum_{j=1}^{M} a_{k j} e^{-i k \theta_{j}}-\sum_{j=M+1}^{L} a_{k j} e^{-i k \theta_{j}}=0, \quad m_{0} \leq k \leq q,
$$

subject to the following conditions:

$$
\begin{aligned}
& a_{k j} \geq 0, \quad m_{0} \leq k \leq q, \quad 1 \leq j \leq L ; \\
& \sum_{j=1}^{L} a_{k j}=1, \quad m_{0} \leq k \leq q ;
\end{aligned}
$$

and

(iii) for $1 \leq j \leq L$, if $a_{k j}=0$

$$
\text { then } a_{k^{\prime} j}=0 \text { for } k<k^{\prime} \leq q \text {. }
$$

If, for every $m_{0}, 0 \leq m_{0} \leq q$, system (1.3) has solutions satisfying conditions (1.4), let $p=0$. Otherwise let $p$ be the largest $m_{0}, 0 \leq m_{0} \leq q$, for which system (1.3) has no solutions satisfying (1.4).

Then for all $f \in \mathscr{M}_{q}(X, Y)$, we have

$$
\lim _{r \rightarrow \infty} \frac{T(r, f)}{r^{p}}=\infty \text { if } p>0,
$$

and

(ii) $\quad \lim _{r \rightarrow \infty} \frac{T(r, f)}{\log r}=\infty$ if $p=0$.

Furthermore, given $\psi(r) \rightarrow \infty$ as $r \rightarrow \infty$, there exists $f \in \mathscr{M}_{q}(X, Y)$ such that

$$
\liminf _{r \rightarrow \infty} \frac{T(r, f)}{\psi(r) r^{p}}=0 \quad \text { if } p>0
$$

and

$$
\text { (ii) } \quad \liminf _{r \rightarrow \infty} \frac{T(r, f)}{\psi(r) \log r}=0 \quad \text { if } p=0 .
$$

Clearly (1.5) asserts that $f \in \mathscr{M}_{q}(X, Y)$ has lower growth at least order $p$, maximal type, and (1.6) asserts that this result is best possible. It is trivial that $p=q$ if $Y=\varnothing$ and $M=1$, giving the result for entire 
functions with zeros on a single ray in [1] and [5]. If $Y=\varnothing$ and $M=2$, an easy verification gives $p \geq \max (0, q-1)$, in agreement with the result in [5] and [11].

A geometric interpretation can be given to the integer $p$ in most cases. Let us suppose that $p \geq 1$ and note that (1.3), (1.4i), and (1.4ii) express the fact that 0 is in the convex hull of $S_{k}$ (defined in (1.2)) for $m_{0} \leq k \leq q$. For $p \geq 1$ we thus have in the cases where we may ignore the rather technical condition (1.4iii) that $p$ is the largest integer $m_{0} \leq q$ for which 0 does not lie in the convex hull of $S_{m_{0}}$.

It would perhaps be helpful to consider an example in which the above geometric interpretation of $p$ fails, i.e. an example in which condition (1.4iii) plays an essential role. Suppose $X=\{0, \pi / 4, \pi / 3\}$, $Y=\varnothing$, and $q=4$. It is elementary that the only solution of (1.3) with $k=4$ subject to (1.4i) and (1.4ii) is

$$
a_{41}=1 / 2, \quad a_{42}=1 / 2, \quad \text { and } \quad a_{43}=0 .
$$

Similarly the only solution of (1.3) with $k=3$ satisfying (1.4i) and (1.4ii) is

$$
a_{31}=1 / 2, \quad a_{32}=0, \text { and } a_{33}=1 / 2 .
$$

There is no solution of (1.3) with $k=2$ subject to (1.4i) and (1.4ii). Thus from (1.4iii), (1.7), and (1.8), it is clear that $p=3$, even though 2 is the largest integer $m_{0}$ not exceeding 4 for which 0 is not in the convex hull of $S_{m_{0}}$.

Although Theorem 1 gives complete information concerning possible lower growth rates of $f \in \mathscr{M}_{q}(X, Y)$ in terms of $q, X$, and $Y$, it does not give information in terms of $q$ and $L$ alone concerning possible lower growth rates of a function of genus $q$ with zeros and poles restricted to any $L$ distinct rays. It would be of interest to determine

$$
\mu(q, L) \equiv \inf p(q, X, Y)
$$

where $X$ and $Y$ vary over all disjoint sets in $[0,2 \pi)$ whose union has $L$ members, and also to consider only entire functions and to determine

$$
\mu_{e}(q, M) \equiv \inf p(q, X, \varnothing)
$$

where $X$ varies over all sets of $M$ members in [0,2 $)$.

From [1], [5], and [11] we have

$$
\mu_{e}(q, M)=\max (0, q-M+1)
$$

for $M=1$ or $M=2$. The possibility of extending (1.9) to other values of $M$ is considered in [11]. In particular it is shown there that if $M$ is a positive integer and $X \subset[0,2 \pi)$ consists of $M$ members, then

$$
\inf _{f \in \mathscr{G}_{q}(X)} \mu(f)=\max (0, q-M+1),
$$


where, for general $X, \mathscr{G}_{q}(X)$ is the subclass of $\mathscr{E}_{q}(X)$ consisting of functions with zeros regularly distributed on each ray, and, for sets $X$ whose members are themselves regularly distributed in $[0,2 \pi), \mathscr{G}_{q}(X)=$ $\mathscr{E}_{q}(X)$.

Theorem 1 shows that (1.9) does not hold in general. Suppose, for example, that $X=\{0, \pi / 180, \pi / 90\}$ and $q=120$. Using Theorem 1 , we have

$$
\mu_{e}(120,3) \leq p(120, X, \varnothing)=90<120-3+1
$$

The quantity $\mu_{e}(q, M)$ has also been studied by E. V. Gleizer. It is my understanding that Gleizer, in a paper [4] submitted to the Ukrainian Journal of Mathematics simultaneously to the submission of this paper, showed

$$
\mu_{e}(q, 3) \geq \max \left(0, \frac{q}{3}-1\right) .
$$

Gleizer also obtained a result for entire functions very close to Theorem 1 applied to $\mathscr{E}_{q}(X)$.

The estimate

$$
\mu_{e}(q, M) \geq\left[\frac{q}{5^{M}}\right]
$$

appears in [2, p. 25]. (The lower growth of entire functions of infinite order with radially distributed zeros is also dealt with in [2, p. 25].) An exact determination of $\mu(q, L)$ and $\mu_{e}(q, M)$ remains open in the general case, as does the probably easier question of whether or not $\mu(q, L)=$ $\mu_{e}(q, L)$.

We also consider the ratio $T(\alpha r, f) / T(r, f)$ for $f \in \mathscr{M}_{q}(X, Y)$.

THEOREM 2. For $\alpha>1$ and $f \in \mathscr{E}_{q}(X)$ of finite order $\lambda$, there exists $K=K(\lambda, \alpha, X)>0$ such that

$$
T(\alpha r, f)<K T(r, f), \quad r>r_{0}(f) .
$$

Theorem 2 generalizes to meromorphic functions in many, but not all, cases. A discussion of the possibility of such a generalization appears in $\S 4$.

It is elementary that (1.10) implies

$$
\lim _{r \rightarrow \infty} \frac{T(r+1, f)}{T(r, f)}=1 \text {. }
$$

(Compare to Corollary 2 of [5].) In [12] it is shown that (1.11) implies that the Nevanlinna deficiency is independent of the choice of the origin. From Theorem 2 we thus conclude that any entire function of finite order for 
which the Nevanlinna deficiency is origin dependent cannot have its zeros restricted to a finite number of rays through any one point. (See for example [8].)

We conclude the Introduction by collecting certain elementary facts needed in the proofs of Theorem 1 and Theorem 2. Our arguments depend heavily on the Fourier series of $\log \left|f\left(\mathrm{re}^{i \theta}\right)\right|$, where $f$ has the form

$$
f(z)=(\exp h(z)) \frac{\Pi_{\nu} E\left(z / z_{\nu}, q\right)}{\Pi E\left(z / w_{\nu}, q\right)}=(\exp h(z)) g(z),
$$

$E(z, q)$ is the Weierstrass factor of genus $q$,

$$
E(z, q)=(1-z) \exp \left(z+z^{2} / 2+\cdots+z^{q} / q\right),
$$

and

$$
h(z)=\sum_{m=1}^{\infty} d_{m} z^{m}, \quad|z|<\infty
$$

Letting

$$
c_{m}(r, f)=\frac{1}{2 \pi} \int_{0}^{2 \pi} e^{-\mathrm{im} \theta} \log \left|f\left(\mathrm{re}^{i \theta}\right)\right| d \theta
$$

we have

(i) $\quad c_{0}(r, f)=N(r, 0)-N(r, \infty)$;

(ii) $\quad c_{m}(r, f)=\overline{c_{-m}(r, f)}, \quad m<0$;

(iii) $\quad c_{m}(r, f)=\frac{d_{m}}{2} r^{m}+c_{m}(r, g)$

$$
\begin{gathered}
=\frac{d_{m}}{2} r^{m}+\frac{1}{2 m}\left\{\sum_{\left|z_{\nu}\right| \leq r}\left(\left(\frac{r}{z_{\nu}}\right)^{m}-\left(\frac{\bar{z}_{\nu}}{r}\right)^{m}\right)\right\} \\
-\frac{1}{2 m}\left\{\sum_{\left|w_{\nu}\right| \leq r}\left(\left(\frac{r}{w_{\nu}}\right)^{m}-\left(\frac{\bar{w}_{\nu}}{r}\right)^{m}\right)\right\}, \\
1 \leq m \leq q ;
\end{gathered}
$$

and

(iv)

$$
\begin{aligned}
& c_{m}(r, f)= \frac{d_{m}}{2} r^{m}+c_{m}(r, g) \\
&= \frac{d_{m}}{2} r^{m}-\frac{1}{2 m}\left\{\sum_{\left|z_{\nu}\right| \leq r}\left(\frac{\bar{z}_{\nu}}{r}\right)^{m}+\sum_{\left|z_{\nu}\right|>r}\left(\frac{r}{z_{\nu}}\right)^{m}\right\} \\
&+\frac{1}{2 m}\left\{\sum_{\left|w_{\nu}\right| \leq r}\left(\frac{\bar{w}_{\nu}}{r}\right)^{m}+\sum_{\left|w_{\nu}\right|>r}\left(\frac{r}{w_{\nu}}\right)^{m}\right\}, \\
& m \geq q+1 .
\end{aligned}
$$


A derivation of these formulas, originally due to F. Nevanlinna [10], can be found in many places, including [9]. Letting $m_{1}(r, f)$ and $m_{2}(r, f)$ denote the $L^{1}$ and $L^{2}$ norms of $\log \left|f\left(\mathrm{re}^{i \theta}\right)\right|$ respectively, we observe trivially from Nevanlinna's first fundamental theorem that for each interger $m$

$$
\frac{\left|c_{m}(r, f)\right|}{2} \leq \frac{m_{1}(r, f)}{2} \leq T(r, f) \leq m_{2}(r, f)+N(r, \infty) .
$$

We shall need the following elementary lemma.

LeMma A. Suppose $m_{1}<m_{2}<\cdots<m_{k}$ and $n_{1}<n_{2}<\cdots<n_{k}$ are nonnegative integers. If $\pi$ is any permutation of $\{1,2, \ldots, k\}$ other than $\pi(j) \equiv k-j+1,1 \leq j \leq k$, then

$$
\sum_{j=1}^{k} m_{j} n_{k-j+1}<\sum_{j=1}^{k} m_{j} n_{\pi(j)} .
$$

Proof. Since $\pi(j) \not \equiv k-j+1$, there exist $1 \leq j_{1}<j_{2} \leq k$ with $\pi\left(j_{1}\right)$ $<\pi\left(j_{2}\right)$. Certainly

$m_{j_{1}} n_{\pi\left(j_{1}\right)}+m_{j_{2}} n_{\pi\left(j_{2}\right)}=m_{j_{1}} n_{\pi\left(j_{2}\right)}+m_{j_{2}} n_{\pi\left(j_{1}\right)}+\left(m_{J_{2}}-m_{j_{1}}\right)\left(n_{\pi\left(j_{2}\right)}-n_{\pi\left(j_{1}\right)}\right)$.

We have

$$
n_{\pi\left(j_{2}\right)}-n_{\pi\left(j_{1}\right)}>0
$$

since $\pi\left(j_{2}\right)>\pi\left(j_{1}\right)$. Since $m_{j_{2}}>m_{j_{1}}$, we conclude

$$
m_{j_{1}} n_{\pi\left(j_{1}\right)}+m_{j_{2}} n_{\pi\left(j_{2}\right)}>m_{j_{1}} n_{\pi\left(j_{2}\right)}+m_{j_{2}} n_{\pi\left(j_{1}\right)},
$$

proving the permutation $\pi$ is not a permutation that minimizes the right side of (1.15).

2. Proof of Theorem 1. We first prove (1.5). Certainly (1.5ii) is trivial by (1.1iii). We thus restrict our attention to the case $p \geq 1$. With no loss in generality we suppose $f(0)=1$. We let $z_{\nu j}$ denote the zeros of $f$ on $\arg z=\theta_{j}, 1 \leq j \leq M$, and let $z_{\nu j}$ denote the poles of $f$ on $\arg z=\theta_{j}$, $M+1 \leq j \leq L$. For $1 \leq j \leq L$ we let $n_{j}(t)$ be the counting function of $\left\{z_{\nu j}\right\}$ and for $p \leq k \leq q$ define

$$
\begin{aligned}
A_{k j}(r) & \equiv \frac{1}{2 k}\left\{\sum_{\left|z_{\nu j}\right| \leq r}\left(\left(\frac{r}{\left|z_{\nu j}\right|}\right)^{k}-\left(\frac{\left|z_{\nu j}\right|}{r}\right)^{k}\right)\right\} \\
& =\frac{1}{2} \int_{0}^{r}\left(\left(\frac{r}{t}\right)^{k}+\left(\frac{t}{r}\right)^{k}\right) \frac{n_{J}(t)}{t} d t .
\end{aligned}
$$


For $0 \leq n \leq q$ we let

$$
C_{n}=\left\{j: 1 \leq j \leq M \text { and } \sum_{\nu} \frac{1}{\left|z_{\nu j}\right|^{n}}<\infty\right\},
$$

(ii) $X_{n}=\left\{\theta_{j}: j \in C_{n}\right\}$,

$$
D_{n}=\left\{j: M+1 \leq j \leq L \text { and } \sum_{\nu} \frac{1}{\left|z_{\nu j}\right|^{n}}<\infty\right\} \text {, }
$$

and

$$
\text { (iv) } \quad Y_{n}=\left\{\boldsymbol{\theta}_{j}: j \in D_{n}\right\} \text {. }
$$

Certainly

$$
X_{n} \subset X_{n+1} \quad \text { and } \quad Y_{n} \subset Y_{n+1}, \quad 0 \leq n \leq q-1 .
$$

We note by (1.1iii) that $X_{q} \cup Y_{q} \subsetneq X \cup Y$.

For $0 \leq n \leq q$, let

$$
p_{n} \equiv p\left(q, X-X_{n}, Y-Y_{n}\right),
$$

where $p(q, \tilde{X}, \tilde{Y})$ is the function defined in the statement of Theorem 1. It follows easily from (2.3) and (2.4) that

$$
p \leq p_{n} \leq p_{n+1} \leq q, \quad 0 \leq n \leq q-1 .
$$

From (2.5) we conclude there exists $n_{0}, p \leq n_{0} \leq q$, such that $p_{n_{0}}=n_{0}$. We select such an $n_{0}$ and set $p^{\prime}=p_{n_{0}}, C^{\prime}=\{1,2, \ldots, M\}-C_{n_{0}}$, $X^{\prime}=X-X_{n_{0}}, D^{\prime}=\{M+1, M+2, \ldots, L\}-D_{n_{0}}$, and $Y^{\prime}=Y-Y_{n_{0}}$. We establish the following lemma.

LEMMA B. The equation

$$
\sum_{j \in C^{\prime}} a_{p^{\prime} j} e^{-i p^{\prime} \theta_{J}}-\sum_{j \in D^{\prime}} a_{p^{\prime} j} e^{-i p^{\prime} \theta_{J}}=0
$$

has no solutions satisfying

$$
a_{p^{\prime} j}>0, \quad j \in C^{\prime} \cup D^{\prime},
$$

and

(ii)

$$
\sum_{j \in C^{\prime} \cup D^{\prime}} a_{p^{\prime} j}=1
$$

Proof of Lemma B. Since $p^{\prime} \geq p \geq 1$, the definition of $p^{\prime}$ implies that $p^{\prime}$ is the largest integer $m_{0} \leq q$ for which the system

$$
\sum_{j \in C^{\prime}} a_{k j} e^{-\imath k \theta_{J}}-\sum_{j \in D^{\prime}} a_{k j} e^{-i k \theta_{J}}=0, \quad m_{0} \leq k \leq q,
$$


has no solutions satisfying

$$
\begin{aligned}
& \text { (i) } \quad a_{k j} \geq 0, \quad j \in C^{\prime} \cup D^{\prime}, m_{0} \leq k \leq q ; \\
& \text { (ii) } \sum_{j \in C^{\prime} \cup D^{\prime}} a_{k_{J}}=1, \quad m_{0} \leq k \leq q
\end{aligned}
$$

and

$$
\begin{aligned}
& \text { (iii) for } j \in C^{\prime} \cup D^{\prime} \text {, if } a_{k j}=0 \\
& \text { then } a_{k^{\prime} j}=0 \text { for } k<k^{\prime} \leq q .
\end{aligned}
$$

If $p^{\prime}=q$, the truth of Lemma $\mathrm{B}$ is immediate from the definition of $p^{\prime}$. If $p^{\prime}<q$, we let

$$
\left\{a_{k j}: p^{\prime}+1 \leq k \leq q, j \in C^{\prime} \cup D^{\prime}\right\}
$$

be a solution of (2.8) with $m_{0}=p^{\prime}+1$ satisfying (2.9). If solutions $\left\{a_{p^{\prime}}\right.$ : $\left.j \in C^{\prime} \cup D^{\prime}\right\}$ of (2.6) exist satisfying (2.7), the combination of $\left\{a_{p^{\prime}}\right.$ : $\left.j \in C^{\prime} \cup D^{\prime}\right\}$ with $\left\{a_{k j}\right\}$ given by (2.10) yields a solution of (2.8) with $m_{0}=p^{\prime}$ satisfying conditions (2.9), including (2.9iii). This contradicts the definition of $p^{\prime}$ and proves Lemma $\mathrm{B}$.

Returning to the proof of Theorem 1 , we conclude from Lemma B that

$$
\tilde{S}_{p^{\prime}} \equiv\left\{e^{-\imath p^{\prime} \theta_{j}}: j \in C^{\prime}\right\} \cup\left\{-e^{-i p^{\prime} \theta_{J}}: j \in D^{\prime}\right\}
$$

lies in a closed halfplane $H$ with boundary line $l$ passing through the origin and that there exists $j_{0} \in C^{\prime} \cup D^{\prime}$ with $e^{-i p^{\prime} \theta_{J_{0}}} \notin l$. If $e^{l \alpha} \in l$ for some real $\alpha$ we have

$$
\sin \left(p^{\prime} \theta_{j_{0}}+\alpha\right) \neq 0
$$

and, since $\tilde{S}_{p^{\prime}} \subset H$,

$$
\begin{gathered}
\left|\sum_{j \in C^{\prime}} A_{p^{\prime} J}(r) e^{-i p^{\prime} \theta_{J}}-\sum_{j \in D^{\prime}} A_{p^{\prime} J}(r) e^{-i p^{\prime} \theta_{J}}\right| . \\
\geq A_{p^{\prime} J_{0}}(r)\left|\sin \left(p^{\prime} \theta_{J_{0}}+\alpha\right)\right|
\end{gathered}
$$

We represent $f$ in form (1.12) and note from (1.13iii) and (2.1) that

$$
c_{p^{\prime}}(r, g)=\sum_{J=1}^{M} A_{p^{\prime} \jmath}(r) e^{-\imath p^{\prime} \theta_{\jmath}}-\sum_{j=M+1}^{L} A_{p^{\prime} J}(r) e^{-i p^{\prime} \theta_{\jmath}} .
$$

From (2.1), (2.2) with $n=n_{0}$, and the fact that $p^{\prime}=n_{0}$, we conclude

$$
A_{p^{\prime} j}=O\left(r^{p^{\prime}}\right), \quad j \notin C^{\prime} \cup D^{\prime},
$$

and

$$
\text { (ii) } \lim _{r \rightarrow \infty} \frac{A_{p^{\prime} j}(r)}{r^{p^{\prime}}}=\infty, \quad j \in C^{\prime} \cup D^{\prime} \text {. }
$$


From (2.11), (2.12), (2.13), and (2.14) we have

$$
\frac{\left|c_{p^{\prime}}(r, g)\right|}{r^{p^{\prime}}} \geq \frac{A_{p^{\prime} j_{0}}(r)\left|\sin \left(p^{\prime} \theta_{J_{0}}+\alpha\right)\right|}{r^{p^{\prime}}}+O(1) \rightarrow \infty
$$

as $r \rightarrow \infty$. From (1.13iii), (1.14), and (2.15) we conclude

$$
\frac{T(r, f)}{r^{p}} \geq \frac{T(r, f)}{r^{p^{\prime}}} \geq \frac{\left|c_{p^{\prime}}(r, f)\right|}{2 r^{p^{\prime}}} \geq \frac{\left|c_{p^{\prime}}(r, g)\right|}{2 r^{p^{\prime}}}-\frac{\left|d_{p^{\prime}}\right|}{4} \rightarrow \infty
$$

as $r \rightarrow \infty$, finishing the proof of (1.5).

We now turn to the proof of (1.6). The case $p=q$ is comparatively simple and we set it aside for later. We take the case $p<q$ and consider system (1.3) with $m_{0}=p+1$ and with solutions $a_{k J}$ satisfying conditions (1.4). Such solutions exist by the definition of $p$. Let

$$
I=\left\{(k, j): p+1 \leq k \leq q, 1 \leq j \leq L, \text { and } a_{k_{J}}>0\right\}
$$

and define

$$
Q \equiv \frac{\max a_{k j}}{\min a_{k j}} \geq 1
$$

where $(k, j)$ varies throughout $I$. Let $\varepsilon>0$ be such that

$$
4 Q(q-p) ! \varepsilon^{1 / 2}<1 .
$$

We select $j, 1 \leq j \leq L$, such that

$$
a_{p+1, j}>0
$$

and define $q^{\prime}=q^{\prime}(j)$ by

$$
q^{\prime} \equiv \max \left\{k: p+1 \leq k \leq q \text { and } a_{k j}>0\right\} .
$$

Thus $q \geq q^{\prime}>p$.

We consider the system of $q^{\prime}-p$ linear equations in $q^{\prime}-p$ unknowns given in matrix form by

$$
A U_{j}=B_{j},
$$

where the $(i, k)$ entry of the $\left(q^{\prime}-p\right) \times\left(q^{\prime}-p\right)$ matrix $A$ is

$$
\varepsilon^{\left(q-q^{\prime}+k-1\right)\left(q^{\prime}-i+1\right)}, \quad 1 \leq i \leq q^{\prime}-p, 1 \leq k \leq q^{\prime}-p,
$$

the entry in the $i$ th row, $1 \leq i \leq q^{\prime}-p$, of the column matrix $U_{J}$ is denoted by $u_{q^{\prime}-i+1}^{0}(j)$, and the entry in the $i$ th row of the column matrix $B_{J}$ is

$$
a_{q^{\prime}-i+1, j} \varepsilon^{-\left(q-q^{\prime}+i-1\right)^{2} / 2}
$$


Our first objective is to show that the (unique) solution $U_{j}$ of (2.20) has all positive entries. Since the only entry of $U$, is clearly positive if $q^{\prime}=p+1$, we temporarily (through equation (2.35)) suppose $q^{\prime}>p+1$.

Certainly the determinant of $A$ is positive. Lemma A and (2.21) imply that among the $\left(q^{\prime}-p\right)$ ! terms comprising $\operatorname{det} A$, the dominant one is the product of the entries on the principal diagonal and that in fact

$$
\begin{aligned}
0 & <1-\left(\left(q^{\prime}-p\right) !-1\right) \varepsilon \leq \frac{\operatorname{det} A}{\varepsilon^{h}} \\
& \leq 1+\left(\left(q^{\prime}-p\right) !-1\right) \varepsilon,
\end{aligned}
$$

where

$$
h=\sum_{i=1}^{q^{\prime}-p}\left(q-q^{\prime}+i-1\right)\left(q^{\prime}-i+1\right) .
$$

We shall use Cramer's Rule to solve for the $k$ th entry $u_{q-k+1}^{0}(j)$ of $U_{J}, 1 \leq k \leq q^{\prime}-p$. For $1 \leq k \leq q^{\prime}-p$, let $A_{k}$ be $A$ with the $k$ th column replaced by $B_{j}$. Thus, by (2.22),

$$
\operatorname{det} A_{k}=\sum_{i=1}^{q^{\prime}-p}(-1)^{i+k} a_{q^{\prime}-\imath+1, j} \varepsilon^{-\left(q-q^{\prime}+\imath-1\right)^{2} / 2} H_{\imath k},
$$

where $H_{i k}$ is the $(i, k)$ minor of $A$.

Let $\varepsilon^{h_{t k}}$ be the largest of the moduli of the $\left(q^{\prime}-p-1\right)$ ! terms of $H_{i k}$. Lemma A implies that if $i \geq k$, then

$$
\begin{aligned}
h_{\imath k}= & \sum_{n=1}^{k-1}\left(q-q^{\prime}+n-1\right)\left(q^{\prime}-n+1\right) \\
& +\sum_{n=k}^{l-1}\left(q-q^{\prime}+n\right)\left(q^{\prime}-n+1\right) \\
& +\sum_{n=i+1}^{q^{\prime}-p}\left(q-q^{\prime}+n-1\right)\left(q^{\prime}-n+1\right),
\end{aligned}
$$

where of course a given sum is omitted if its lower limit of summation exceeds its upper limit (for instance the second sum if $i=k$ or the third sum if $i=q^{\prime}-p$ ). Elementary algebra leads from (2.25) to

$$
h_{i k}=D\left(q, q^{\prime}, k, p\right)+\frac{i^{2}}{2}-\frac{i}{2}+i\left(q-q^{\prime}\right)
$$

for some function $D\left(q, q^{\prime}, k, p\right)$ independent of $i$. 
Similarly, for $i \leq k$, we have by Lemma A

$$
\begin{aligned}
h_{i k}= & \sum_{n=1}^{i-1}\left(q-q^{\prime}+n-1\right)\left(q^{\prime}-n+1\right) \\
& +\sum_{n=i+1}^{k}\left(q-q^{\prime}+n-2\right)\left(q^{\prime}-n+1\right) \\
& +\sum_{n=k+1}^{q^{\prime}-p}\left(q-q^{\prime}+n-1\right)\left(q^{\prime}-n+1\right) \\
= & D\left(q, q^{\prime}, k, p\right)+k+\frac{i^{2}}{2}-\frac{3 i}{2}+i\left(q-q^{\prime}\right) .
\end{aligned}
$$

Direct calculation from (2.26) and (2.27) shows for $i \geq k$ that

$$
h_{\imath k}-\frac{1}{2}\left(q-q^{\prime}+i-1\right)^{2}=D_{1}\left(q, q^{\prime}, k, p\right)+i / 2
$$

for some function $D_{1}\left(q, q^{\prime}, k, p\right)$ independent of $i$ and for $i \leq k$ that

$$
h_{i k}-\frac{1}{2}\left(q-q^{\prime}+i-1\right)^{2}=D_{1}\left(q, q^{\prime}, k, p\right)+k-i / 2 .
$$

From (2.28) and (2.29) we conclude for $1 \leq k \leq q^{\prime}-p$ that

$$
\begin{aligned}
\frac{1}{2}+h_{k k} & -\frac{1}{2}\left(q-q^{\prime}+k-1\right)^{2} \\
= & \min _{\substack{1 \leq i \leq q^{\prime}-p \\
i \neq k}}\left(h_{i k}-\frac{1}{2}\left(q-q^{\prime}+i-1\right)^{2}\right) .
\end{aligned}
$$

Certainly for $1 \leq i \leq q^{\prime}-p$ and $1 \leq k \leq q^{\prime}-p$ we have

$$
\left|H_{i k}\right| \leq\left(q^{\prime}-p-1\right) ! \varepsilon^{h_{t k}}
$$

and thus by (2.30) for $1 \leq i \leq q^{\prime}-p, i \neq k$,

$$
\varepsilon^{-\left(q-q^{\prime}+i-1\right)^{2} / 2}\left|H_{i k}\right| \leq\left(q^{\prime}-p-1\right) ! \varepsilon^{1 / 2+h_{k k}-\left(q-q^{\prime}+k-1\right)^{2} / 2} .
$$

From (2.16), (2.17), and (2.31) we conclude for $1 \leq k \leq q^{\prime}-p$ that

$$
\begin{aligned}
& \left|\sum_{\substack{i=1 \\
i \neq k}}^{q^{\prime}-p}(-1)^{i+k} a_{q-i+1, j} \varepsilon^{-\left(q-q^{\prime}+t-1\right)^{2} / 2} H_{i k}\right| \\
& \quad \leq\left(q^{\prime}-p\right) ! Q a_{q^{\prime}-k+1, j} \varepsilon^{1 / 2+h_{k k}-\left(q-q^{\prime}+k-1\right)^{2} / 2} \\
& \quad<a_{q^{\prime}-k+1, j} \varepsilon^{h_{k k}-\left(q-q^{\prime}+k-1\right)^{2} / 2} / 4 .
\end{aligned}
$$


The reasoning leading to (2.23), applied to $H_{k k}$ rather than $\operatorname{det} A$, yields

$$
\begin{aligned}
\frac{1}{2} & <1-\left(\left(q^{\prime}-p-1\right) !-1\right) \varepsilon \\
& \leq \frac{H_{k k}}{\varepsilon^{h_{k k}}} \leq 1+\left(\left(q^{\prime}-p-1\right) !-1\right) \varepsilon .
\end{aligned}
$$

Upon combining (2.24), (2.32), and (2.33), we conclude

$$
\operatorname{det} A_{k}>a_{q^{\prime}-k+1, j} \varepsilon^{h_{k k}-\left(q-q^{\prime}+k-1\right)^{2} / 2} / 4>0 .
$$

Cramer's Rule in conjunction with (2.23) and (2.34) thus yields

$$
u_{q^{\prime}-k+1}^{0}(j)=\frac{\operatorname{det} A_{k}}{\operatorname{det} A}>0, \quad 1 \leq k \leq q^{\prime}-p .
$$

Certainly this conclusion also holds in the trivial case $q^{\prime}=p+1$, when (2.20) is a $1 \times 1$ system. We remark that an examination of (2.23), (2.24), (2.32), and (2.33) shows that for small $\varepsilon>0$ the solution of (2.20) is approximately the solution of the system (2.20) with $A$ modified so that its entries off the principal diagonal are 0.

We next modify the linear system (2.20) in such a way that the solutions are in fact positive integers. For $p+1 \leq m \leq q^{\prime}$ we consider the system of equations

$$
\begin{aligned}
& F_{m}\left(b_{1}, b_{2}, \ldots, b_{q^{\prime}-p}, u_{q^{\prime}}, u_{q^{\prime}-1}, \ldots, u_{p+1}\right) \\
& \equiv \sum_{k=1}^{q^{\prime}-p} b_{k}^{m} u_{q^{\prime}-k+1}-a_{m, j} \varepsilon^{-(q-m)^{2} / 2}=0 .
\end{aligned}
$$

We do not indicate the dependence of $F_{m}$ upon $j$ in the notation.

We let $P_{0}(j)$ be the point in $2\left(q^{\prime}-p\right)$ dimensional Euclidean space given by

$$
P_{0}(j)=\left(\varepsilon^{q-q^{\prime}}, \varepsilon^{q-q^{\prime}+1}, \ldots, \varepsilon^{q-p-1}, u_{q^{\prime}}^{0}(j), u_{q^{\prime}-1}^{0}(j), \ldots, u_{p+1}^{0}(j)\right) .
$$

From (2.20) we have

$$
F_{m}\left(P_{0}(j)\right)=0, \quad p+1 \leq m \leq q^{\prime} .
$$

We also have

$$
\left.\frac{\partial\left(F_{p+1}, F_{p+2}, \ldots, F_{q^{\prime}}\right)}{\partial\left(b_{1}, b_{2}, \ldots, b_{q^{\prime}-p}\right)}\right|_{P_{0}(j)}=\frac{q^{\prime} !}{p !} u_{q^{\prime}}^{0}(j) \cdots u_{p+1}^{0}(j) \Delta,
$$


where $\Delta$ is the determinant of the $\left(q^{\prime}-p\right) \times\left(q^{\prime}-p\right)$ matrix whose $(i, k)$ entry is $b_{k}^{p+i-1}$ with

$$
b_{k}=\varepsilon^{q-q^{\prime}+k-1}
$$

Evidently we have

$$
\Delta=\left(\prod_{k=1}^{q^{\prime}-p} b_{k}^{p}\right) V \neq 0,
$$

where $V$ is the van der Monde determinant associated with the distinct numbers $b_{k}, 1 \leq k \leq q^{\prime}-p$.

In view of (2.37) and (2.38), we may apply the Implicit Function Theorem to assert the existence of $\delta>0$ independent of $j$, a cube $E_{j}$ of side $\delta$ in $q^{\prime}-p$ dimensional Euclidean space centered at

$$
\left(u_{q^{\prime}}^{0}(j), u_{q^{\prime}-1}^{0}(j), \ldots, u_{p+1}^{0}(j)\right)
$$

and positive $C^{1}$ functions $\varphi_{1}, \varphi_{2}, \ldots, \varphi_{q^{\prime}-p}$ defined on $E_{j}$ such that if $p+1 \leq m \leq q^{\prime}$, then

$$
\begin{aligned}
F_{m}\left(\varphi_{1}\left(u_{q^{\prime}}, \ldots, u_{p+1}\right), \varphi_{2}\left(u_{q^{\prime}}, \ldots, u_{p+1}\right), \ldots,\right. \\
\left.\varphi_{q^{\prime}-p}\left(u_{q^{\prime}}, \ldots, u_{p+1}\right), u_{q^{\prime}}, u_{q^{\prime}-1}, \ldots, u_{p+1}\right) \equiv 0
\end{aligned}
$$

for $\left(u_{q^{\prime}}, u_{q^{\prime}-1}, \ldots, u_{p+1}\right) \in E_{j}$.

For a positive integer $\nu$, let $R_{\nu}>0$ independent of $j$ be such that

$$
\delta R_{\nu}^{p+1}>1 \text {. }
$$

Select $\beta \in(0,1)$ and then let $\left(u_{q^{\prime}}, u_{q^{\prime}-1}, \ldots, u_{p+1}\right) \in E_{j}$ be such that

$$
n_{q^{\prime}-k+1}=R_{\nu}^{q^{\prime}+\beta} u_{q^{\prime}-k+1}, \quad 1 \leq k \leq q^{\prime}-p,
$$

is a positive integer. This choice is possible by $(2.40)$.

Let

$$
\alpha_{k}=\varphi_{k}\left(u_{q^{\prime}}, u_{q^{\prime}-1}, \ldots, u_{p+1}\right), \quad 1 \leq k \leq q^{\prime}-p .
$$

Let $g_{j}$ be the Weierstrass product of genus $q^{\prime}$ having a zero of multiplicity $n_{q^{\prime}-k+1}$ at $t_{k} e^{i \theta_{j}}$, where

$$
t_{k}=R_{\nu} \alpha_{k}^{-1}, \quad 1 \leq k \leq q^{\prime}-p .
$$

(We suppress the dependence of $g_{j}$ on $\nu$ in the notation as well as the dependence of $n_{q^{\prime}-k+1}$ and $t_{k}$ on both $j$ and $\nu$.) 
For $p+1 \leq m \leq q^{\prime}$, we calculate the quantity

$$
\begin{aligned}
c_{m j} & \equiv \sum_{k=1}^{q^{\prime}-p} \frac{n_{q^{\prime}-k+1}}{t_{k}^{m}}=R_{\nu}^{q^{\prime}+\beta-m} \sum_{k=1}^{q^{\prime}-p} \alpha_{k}^{m} u_{q^{\prime}-k+1} \\
& =R_{\nu}^{q^{\prime}+\beta-m} a_{m, j} \varepsilon^{-(q-m)^{2} / 2},
\end{aligned}
$$

where in the first step we use (2.41) and (2.43) and in the second step we use (2.36), (2.39), and (2.42).

From (1.13iii) and (2.44) for all $r>t_{k}=t_{k}(j), 1 \leq k \leq q^{\prime}-p$, we have for $p+1 \leq m \leq q^{\prime}$,

$$
\begin{aligned}
c_{m}\left(r, g_{j}\right) & =\frac{r^{m}}{2 m} c_{m j} e^{-i m \theta_{J}}+O\left(n\left(r, 0, g_{J}\right)\right) \\
& =\frac{R_{\nu}^{q^{\prime}+\beta}}{2 m}\left(\frac{r}{R_{\nu}}\right)^{m} a_{m, j} \varepsilon^{-(q-m)^{2} / 2} e^{-\imath m \theta_{J}}+O\left(n\left(r, 0, g_{j}\right)\right) .
\end{aligned}
$$

From (1.13iv), (2.19), and (2.45) we see that in fact

$$
\begin{aligned}
c_{m}\left(r, g_{j}\right)= & \frac{R_{\nu}^{q^{\prime}+\beta}}{2 m}\left(\frac{r}{R_{\nu}}\right)^{m} a_{m, j} \varepsilon^{-(q-m)^{2} / 2} e^{-i m \theta_{j}} \\
& +O\left(n\left(r, 0, g_{j}\right)\right)
\end{aligned}
$$

for $r>t_{k}(j), 1 \leq k \leq q^{\prime}-p$, for all $m, p+1 \leq m \leq q$, and for all $j$ satisfying (2.18).

For $j$ not satisfying (2.18), we let $g_{j}=1$. Thus (2.46) holds for all $j$, $1 \leq j \leq L$, all $m, p+1 \leq m \leq q$, and all large $r$.

Recalling that $g_{j}$ in general depends on $\nu$, we define

$$
f_{\nu}=\prod_{j=1}^{M} g_{j} / \prod_{j=M+1}^{L} g_{j} .
$$

Letting $n\left(r, f_{\nu}\right)=n\left(r, 0, f_{\nu}\right)+n\left(r, \infty, f_{\nu}\right)$, we then have by (2.46) for all large $r$ and $p+1 \leq m \leq q$,

$$
\begin{aligned}
c_{m}\left(r, f_{\nu}\right)= & \frac{R_{\nu}^{q^{\prime}+\beta}}{2 m}\left(\frac{r}{R_{\nu}}\right)^{m} \varepsilon^{-(q-m)^{2} / 2} \\
& \cdot\left\{\sum_{j=1}^{M} a_{m j} e^{-i m \theta_{j}}-\sum_{j=M+1}^{L} a_{m j} e^{-i m \theta_{j}}\right\}+O\left(n\left(r, f_{\nu}\right)\right) .
\end{aligned}
$$

From (1.3) we conclude for large $r$ that

$$
c_{m}\left(r, f_{\nu}\right)=O\left(n\left(r, f_{\nu}\right)\right) \leq \frac{r^{p}(\psi(r))^{1 / 2}}{8 \sqrt{q} \nu^{2}}, \quad p+1 \leq m \leq q .
$$


We now suppose $p \geq 1$ and let

$$
A_{p}\left(r, f_{\nu}\right)=\frac{1}{2} \int_{0}^{r}\left(\left(\frac{r}{t}\right)^{p}+\left(\frac{t}{r}\right)^{p}\right) \frac{n\left(t, f_{\nu}\right)}{t} d t .
$$

(Compare to (2.1).) From (1.13iii) we have for $1 \leq m \leq p$ and sufficiently large $r$

$$
\left|c_{m}\left(r, f_{\nu}\right)\right| \leq A_{p}\left(r, f_{\nu}\right) \leq r^{p}(\psi(r))^{1 / 2} / 8 \sqrt{p} \nu^{2} .
$$

From (1.13iv) we have

$$
\left|c_{m}\left(r, f_{\nu}\right)\right| \leq \frac{n\left(r, f_{\nu}\right)}{2 m} \leq \frac{r^{p}(\psi(r))^{1 / 2}}{8 m \nu^{2}}
$$

for $m \geq q+1$ and sufficiently large $r$. Certainly

$$
N\left(r, f_{\nu}\right)<r^{p}(\psi(r))^{1 / 2} / 8 \nu^{2}
$$

for large $r$. From Parseval's formula, (1.14), (2.47), (2.48), (2.49), and (2.50) we have

$$
T\left(r, f_{\nu}\right) \leq N\left(r, f_{\nu}\right)+m_{2}\left(r, f_{\nu}\right)<\frac{r^{p}(\psi(r))^{1 / 2}}{2 \nu^{2}}
$$

for sufficiently large $r$.

The proof in the case $0<p<q$ is completed by taking

$$
f=\prod_{\nu=1}^{\infty} f_{\nu}
$$

where $f_{\nu}$ is a function of the sort just constructed and the sequence $R_{\nu}$ tends to infinity very rapidly. (The product converges by $(2.41)$ and (2.43).) We consider a sequence $r_{\nu} \rightarrow \infty$ such that

$$
R_{\nu} \ll r_{\nu} \ll R_{\nu+1} \text {. }
$$

If the $R_{\nu}$ 's are sufficiently widely spaced, we easily calculate from (1.13iv) that

$$
T\left(r_{\nu}, \prod_{k=\nu+1}^{\infty} f_{k}\right) \leq m_{2}\left(r_{\nu}, \prod_{k=\nu+1}^{\infty} f_{k}\right) \leq 1 .
$$

From (2.51) we have (since $r_{\nu}>R_{\nu}$ ) that

$$
T\left(r_{\nu}, \prod_{k=1}^{\nu} f_{k}\right) \leq \sum_{k=1}^{\nu} T\left(r_{\nu}, f_{k}\right)+\log \nu<r_{\nu}^{p}\left(\psi\left(r_{\nu}\right)\right)^{1 / 2} .
$$

The combination of (2.52) with (2.53) completes the proof of (1.6i) in the case $p<q$. 
In the case $0=p<q$, the discussion following (2.47) applies with only the trivial modifications that (2.48) is omitted, $r^{p}$ is replaced by $\log r$ in (2.50) and (2.51), and $r_{\nu}^{p}$ is replaced by $\log r_{\nu}$ in (2.53). This proves (1.6ii) in the case $p<q$.

The construction is much simpler if $p=q$. We assume without loss of generality that $X \neq \varnothing$. In this case $f$ can in fact be taken to be entire with zeros only on the ray $\arg z=\theta_{1} \in X$. We choose a sequence $R_{\nu}$ increasing rapidly to infinity. We select $\beta \in(0,1)$ and let $f_{\nu}$ be the $\left[R_{\nu}^{q+\beta}\right]$ power of the Weierstrass factor of genus $q$ with zero at $R_{\nu} e^{i \theta_{1}}$. If $p>0$, the discussion from (2.48) through (2.53) applies to yield (1.6i). Note this case is far simpler than the $p<q$ case since no reference need be made to (2.47). Finally, if $0=p=q$, we again omit (2.48), replace $r^{p}$ by $\log r$ in (2.50) and (2.51), and replace $r_{v}^{p}$ by $\log r_{v}$ in (2.53). This completes the proof of Theorem 1.

An examination of the proof of (1.6) shows the function we have constructed has order $q+\beta$ where $0<\beta<1$. By letting $\beta$ vary with $\nu$, a function of any order in $[q, q+1]$ can be produced satisfying (1.6).

3. Proof of Theorem 2. Without loss of generality we may presume $\alpha=2$ and $f(0)=1$. It follows from a theorem of Weyl [13, Satz 16] that there exists $p>\lambda \geq q$ such that

$$
\cos p \theta_{j}>\sqrt{1 / 2}, \quad 1 \leq j \leq M .
$$

Details of the argument establishing the existence of such a $p$ appear in [3] or [7]. As before we let $\left\{z_{\nu}\right\}$ denote the zeros of $f$ and write $n(r)=n(r, 0)$. We represent $f$ in the form

$$
f(z)=(\exp h(z)) \prod_{\nu} E\left(\frac{z}{z_{\nu}}, q\right),
$$

where the polynomial $h$ is given by

$$
h(z)=\sum_{m=1}^{k} d_{m} z^{m} .
$$

If $k \geq q+1$ and $d_{k} \neq 0$, it is elementary that

$$
T(r, f) \sim \frac{\left|d_{k}\right|}{\pi} r^{k}=\frac{\left|d_{k}\right|}{\pi} r^{\lambda},
$$

implying

$$
T(2 r, f)<2^{\lambda+1} T(r, f), \quad r>r_{0}(f) .
$$

Thus we suppose $k \leq q$. 
From (1.13iii) and (1.13iv) we have

(i)

$$
\begin{aligned}
c_{m}\left(\frac{r}{2}, f\right)= & \frac{d_{m}}{2}\left(\frac{r}{2}\right)^{m} \\
+ & \frac{1}{2 m}\left\{\sum_{\left|z_{\nu}\right| \leq r / 2}\left(\left(\frac{r}{2 z_{\nu}}\right)^{m}-\left(\frac{2 \bar{z}_{\nu}}{r}\right)^{m}\right)\right\}, \\
& 1 \leq m \leq q ;
\end{aligned}
$$

(ii)

$$
\begin{aligned}
c_{m}(2 r, f)= & \frac{d_{m}}{2}(2 r)^{m} \\
& +\frac{1}{2 m}\left\{\sum_{\left|z_{\nu}\right| \leq 2 r}\left(\left(\frac{2 r}{z_{\nu}}\right)^{m}-\left(\frac{\bar{z}_{\nu}}{2 r}\right)^{m}\right)\right\}, \\
& 1 \leq m \leq q ;
\end{aligned}
$$

(iii)

$$
\begin{array}{r}
c_{m}\left(\frac{r}{2}, f\right)=-\frac{1}{2 m}\left\{\sum_{\left|z_{\nu}\right| \leq r / 2}\left(\frac{2 \bar{z}_{\nu}}{r}\right)^{m}+\sum_{\left|z_{\nu}\right|>r / 2}\left(\frac{r}{2 z_{\nu}}\right)^{m}\right\}, \\
m \geq q+1 ;
\end{array}
$$

and

(iv)

$$
\begin{array}{r}
c_{m}(2 r, f)=-\frac{1}{2 m}\left\{\sum_{\left|z_{\nu}\right| \leq 2 r}\left(\frac{\bar{z}_{\nu}}{2 r}\right)^{m}+\sum_{\left|z_{\nu}\right|>2 r}\left(\frac{2 r}{z_{\nu}}\right)^{m}\right\} \\
m \geq q+1 .
\end{array}
$$

Critical to our argument is the following inequality (3.4), which bounds the number of zeros near $|z|=r$ in terms of $T(r, f)$. We have, by (1.14), (3.1), and (3.3iii),

$$
\begin{aligned}
\frac{2^{-p}}{2 p}\left(n(2 r)-n\left(\frac{r}{2}\right)\right) \leq \frac{1}{2 p} \sum_{r / 2<\left|z_{\nu}\right| \leq 2 r}\left(\frac{r}{\left|z_{\nu}\right|}\right)^{p} \\
\leq \frac{1}{2 p} \sum_{\left|z_{\nu}\right|>r / 2}\left(\frac{r}{\left|z_{\nu}\right|}\right)^{p}<\frac{1}{p}\left|\sum_{\left|z_{\nu}\right|>r / 2}\left(\frac{r}{z_{\nu}}\right)^{p}\right| \\
=2\left|2^{p} c_{p}\left(\frac{r}{2}, f\right)+\frac{4^{p}}{2 p} \sum_{\left|z_{\nu}\right| \leq r / 2}\left(\frac{\bar{z}_{\nu}}{r}\right)^{p}\right| \\
\leq 2^{p+1}\left|c_{p}\left(\frac{r}{2}, f\right)\right|+2^{p} n\left(\frac{r}{2}\right) \\
\leq 2^{p+3} T(r, f) .
\end{aligned}
$$

We conclude that

$$
n(2 r)-n(r / 2) \leq p 2^{2 p+4} T(r, f) .
$$


Since $n(r / 2) \leq 2 T(r, f)$, we see that in fact

$$
n(2 r)<p 2^{2 p+5} T(r, f) .
$$

Using (3.3i) we have for $1 \leq m \leq q$ and $r>0$

$$
\begin{gathered}
4^{-m}\left(\frac{d_{m}}{2}(2 r)^{m}+\frac{1}{2 m} \sum_{\left|z_{\nu}\right| \leq r / 2}\left(\frac{2 r}{z_{\nu}}\right)^{m}\right) \\
=\frac{d_{m}}{2}\left(\frac{r}{2}\right)^{m}+\frac{1}{2 m} \sum_{\left|z_{\nu}\right| \leq r / 2}\left(\frac{r}{2 z_{\nu}}\right)^{m} \\
=c_{m}\left(\frac{r}{2}, f\right)+\frac{1}{2 m} \sum_{\left|z_{\nu}\right| \leq r / 2}\left(\frac{2 \bar{z}_{\nu}}{r}\right)^{m} .
\end{gathered}
$$

We conclude from (1.14), (3.3ii), (3.5), and (3.6) that for $1 \leq m \leq q$ and $r>0$

$$
\begin{aligned}
\left|c_{m}(2 r, f)\right| & \leq\left|\frac{d_{m}}{2}(2 r)^{m}+\frac{1}{2 m} \sum_{\left|z_{\nu}\right| \leq 2 r}\left(\frac{2 r}{z_{\nu}}\right)^{m}\right|+\frac{n(2 r)}{m} \\
& \leq 4^{m}\left|c_{m}\left(\frac{r}{2}, f\right)\right|+\frac{4^{m}}{2 m} n\left(\frac{r}{2}\right)+p 2^{2 p+5} T(r, f) \\
& \leq\left(2^{2 m+2}+p 2^{2 p+5}\right) T(r, f) \leq p 2^{2 p+6} T(r, f) .
\end{aligned}
$$

We next consider $m \geq q+1$ and let

$$
B=B(r, m)=-\frac{1}{2 m} \sum_{\left|z_{\nu}\right|>2 r}\left(\frac{2 r}{z_{\nu}}\right)^{m} .
$$

We distinguish two cases. First suppose $p+1 \leq m$. From (1.14), (3.1), and (3.3iii) we conclude

$$
\begin{aligned}
2 m|B| & \leq \sum_{\left|z_{\nu}\right|>2 r}\left(\frac{2 r}{\left|z_{\nu}\right|}\right)^{p} \\
& \leq 2^{2 p+1}\left|\sum_{\left|z_{\nu}\right|>2 r}\left(\frac{r}{2 z_{\nu}}\right)^{p}\right| \leq 2^{2 p+1}\left|\sum_{\left|z_{\nu}\right|>r / 2}\left(\frac{r}{2 z_{\nu}}\right)^{p}\right| \\
& =2^{2 p+1}\left|2 p c_{p}\left(\frac{r}{2}, f\right)+\sum_{\left|z_{\nu}\right| \leq r / 2}\left(\frac{2 \bar{z}_{\nu}}{r}\right)^{p}\right| \\
& \leq 2^{2 p+1}(4 p T(r, f)+n(r / 2))<p 2^{2 p+4} T(r, f) .
\end{aligned}
$$

Next suppose $q+1 \leq m \leq p$. We have

$$
B=-\frac{1}{2 m} \sum_{\left|z_{\nu}\right|>r / 2}\left(\frac{2 r}{z_{\nu}}\right)^{m}+\frac{1}{2 m} \sum_{r / 2<\left|z_{\nu}\right| \leq 2 r}\left(\frac{2 r}{z_{\nu}}\right)^{m}=B_{1}+B_{2} \text {. }
$$


Certainly

$$
\begin{aligned}
4^{-m}\left|B_{1}\right| & =\left|c_{m}\left(\frac{r}{2}, f\right)+\frac{1}{2 m} \sum_{\left|z_{\nu}\right| \leq r / 2}\left(\frac{2 \bar{z}_{\nu}}{r}\right)^{m}\right| \\
& \leq 2 T(r, f)+n(r / 2) / 2 m \leq 3 T(r, f) .
\end{aligned}
$$

By (3.4) we have

$$
\left|B_{2}\right|=\frac{4^{p}}{2 m}\left(n(2 r)-n\left(\frac{r}{2}\right)\right) \leq \frac{p}{m} 2^{4 p+3} T(r, f) .
$$

Combining (3.9) and (3.10) we conclude for $q+1 \leq m \leq p$ that

$$
|B| \leq \frac{p}{m} 2^{4 p+4} T(r, f) \text {. }
$$

From (3.8) and (3.11) we have for all $m \geq q+1$ that

$$
|B| \leq \frac{p}{m} 2^{4 p+4} T(r, f) .
$$

From (3.3iv), (3.5), and (3.12) we conclude

$$
\left|c_{m}(2 r, f)\right| \leq|B|+\frac{n(2 r)}{2 m} \leq \frac{p}{m} 2^{4 p+5} T(r, f)
$$

for $m \geq q+1$ and $r>0$.

Certainly for $r>0$ by (3.5)

$$
\begin{aligned}
N(2 r) & =N(r)+(N(2 r)-N(r)) \\
& \leq T(r, f)+n(2 r) \leq p 2^{2 p+6} T(r, f) .
\end{aligned}
$$

From (3.7), (3.13), and (3.14) we conclude

$$
\begin{aligned}
& m_{2}(2 r, f)^{2}=\sum_{m=-\infty}^{\infty}\left|c_{m}(2 r, f)\right|^{2} \\
& \quad \leq\left(p^{2} 2^{4 p+12}+2 q p^{2} 2^{4 p+12}+4 p^{2} 2^{8 p+10}\right) T(r, f)^{2} .
\end{aligned}
$$

Since $T(2 r, f) \leq m_{2}(2 r, f)$ for the entire function $f,(1.10)$ follows from (3.2) and (3.15) with

$$
K=K(\lambda, 2, X)=\max \left(2^{\lambda+1}, p 2^{4 p+5}(5+2 \lambda)^{1 / 2}\right) .
$$

We observe that $p$ depends on $\lambda$ and $X$, as in turn does the entire right side of (3.16). This completes the proof of Theorem 2 .

4. Concluding remarks. The conclusion of Theorem 2 holds for the class $\mathscr{M}_{q}(X, Y)$ provided the numbers $\theta_{1}, \theta_{2}, \ldots, \theta_{L}$ are linearly independent over the integers. It follows in this case from Weyl's theorem [13, Satz 16] that there exists $p>\lambda$ such that

$$
\cos p \theta_{j}>\sqrt{1 / 2}, \quad 1 \leq j \leq M,
$$


and

$$
\cos p \theta_{j}<-\sqrt{1 / 2}, \quad M+1 \leq j \leq L .
$$

The proof given in $\$ 3$ may be adapted in this situation to $f \in \mathscr{M}_{q}(X, Y)$ with only trivial modifications.

If $X \cup Y$ is linearly dependent over the integers, the conclusion of Theorem 2 may fail for the class $\mathscr{M}_{q}(X, Y)$. For example, let $X=\left\{\theta_{1}\right\}$ where $\theta_{1}=0$ and let $Y=\left\{\theta_{2}, \theta_{3}, \theta_{4}, \theta_{5}\right\}$ where $\theta_{j}=2 \pi(j-1) / 5,2 \leq j$ $\leq 5$. Trivially there exist $a_{k j}>0$ for $1 \leq j \leq 5$ and all positive integers $k$ such that

$$
a_{k 1} e^{-i k \theta_{1}}-\sum_{j=2}^{5} a_{k j} e^{-i k \theta_{j}}=0 .
$$

Suppose $q$ and $J_{n}$ are arbitrary integers subject only to the condition $1 \leq q \leq J_{n}$. By a construction based on our proof of (1.6), we may produce $R_{n} \rightarrow \infty, \beta_{n} \rightarrow \infty$, and

$$
f_{n}(z)=\prod_{\nu} E\left(\frac{z}{z_{\nu}}, q\right) / \prod_{\nu} E\left(\frac{z}{w_{\nu}}, q\right)
$$

having the following properties:

$$
\begin{aligned}
\text { (i) } & \arg z_{\nu} \in X, \\
\text { (ii) } & \arg w_{\nu} \in Y, \\
\text { (iii) } & R_{n} \leq\left|z_{\nu}\right| \leq \beta_{n} R_{n}, \\
\text { (iv) } & R_{n} \leq\left|w_{\nu}\right| \leq \beta_{n} R_{n}, \\
\text { (v) } & c_{m}\left(R_{n}, f_{n}\right)=0, \quad q+1 \leq m \leq J_{n},
\end{aligned}
$$

and

$$
\text { (vi) } \quad\left|c_{m}\left(R_{n}, f_{n}\right)\right| \leq \frac{n\left(2 R_{n}, f_{n}\right)}{m}, \quad m>J_{n},
$$

where $n\left(r, f_{n}\right)=n\left(r, 0, f_{n}\right)+n\left(r, \infty, f_{n}\right)$.

Only minor adaptations of the construction of the $f_{\nu}$ 's used in the proof of (1.6) are needed to produce $f_{n}$ 's satisfying (4.3). In the present context, $J_{n}$ plays the role of $q$ in the proof of (1.6) and $q+1$ plays the role of $p+1$. The careful placement (using (4.1) for $q+1 \leq k \leq J_{n}$ ) of the $z_{\nu}$ 's and $w_{\nu}$ 's as in the proof of (1.6) yields (4.3v); rough estimates on the resulting function $n\left(t, f_{n}\right)$ combined with (1.13iv) yield (4.3vi).

From (1.13iii), (4.3iii), and (4.3iv) it is immediate that

$$
c_{m}\left(R_{n}, f_{n}\right)=0, \quad 0 \leq m \leq q .
$$

From (4.3) and (4.4) we have

$$
T\left(R_{n}, f_{n}\right) \leq m_{2}\left(R_{n}, f_{n}\right) \leq \frac{n\left(2 R_{n}, f_{n}\right)}{J_{n}^{1 / 2}} .
$$


Trivially we have

$$
n\left(2 R_{n}, f_{n}\right)<4 T\left(4 R_{n}, f_{n}\right) .
$$

Finally we produce $f \in \mathscr{M}_{q}(X, Y)$ by setting

$$
f=\prod_{n=1}^{\infty} f_{n}
$$

where the $f_{n}$ 's are associated with a widely spaced sequence $R_{n}$ and $J_{n}$ tends to infinity. Using (4.5) and (4.6) we are able to conclude

$$
\limsup _{r \rightarrow \infty} \frac{T(2 r, f)}{T(r, f)}=\infty .
$$

We omit the rather lengthy details of this argument.

\section{REFERENCES}

[1] A. Edrei and W. H. J. Fuchs, On the growth of meromorphic functions with several deficient values, Trans. Amer. Math. Soc., 93 (1959), 292-328.

[2] - On the maximum number of deficient values of certain classes of functions, Air Force Technical Note, AFOSR TN 60-402 (April 1960).

[3] A. Edrei, W. H. J. Fuchs, and S. Hellerstein, Radial distribution and deficiencies of the values of a meromorphic function, Pacific J. Math., 11 (1961), 135-151.

[4] E. V. Gleizer, preprint submitted to Ukr. Matemat. Zhurnal.

[5] A. A. Gol'dberg, Meromorphic functions with separated zeros and poles, (in Russian), Izv. Vysš. Učebn. Zaved. Matemat., 17, no. 4 (1960), 67-72.

[6] A. A. Gol'dberg and I. V. Ostrovskii, The distribution of values of meromorphic functions, (in Russian) (Izdat. Nauk, Moscow, 1970).

[7] J. Miles, On entire functions of infinite order with radially distributed zeros, Pacific J. Math., 81 (1979), 131-157.

[8] Some examples of the dependence of the Nevanlinna deficiency upon the choice of origin, Proc. London Math. Soc., (3), 47 (1983), 145-176.

[9] J. Miles and D. F. Shea, An extremal problem in value distribution theory, Quart. J. Math. Oxford (2), 24 (1973), 458-464.

[10] F. Nevanlinna, Bemerkungen zur Theorie der ganzen Funktionen endlicher Ordnung, Soc. Sci. Fenn. Comment. Phys.-Math., 2 Nr. 4 (1923), 1-7.

[11] N. Steinmetz, On the order and lower order of entire functions with radially distributed zeros, Proc. Amer. Math. Soc., 87, no. 3 (1983), 449-452.

[12] G. Valiron, Valeurs exceptionelles et valeurs déficientes des fonctions méromorphes, C. R. Acad. Sci. Paris, 225 (1947), 556-558.

[13] H. Weyl, Über die Gleichverteilung von Zahlen mod. Eins, Math. Annalen, 77 (1916), 313-352.

Received September 5, 1984. The author wishes to thank the University of Hawaii for its hospitality during the preparation of this paper.

UNIVERSITY OF ILLINOIS AT URBANA-CHAMPAIGN

1409 West Green STreet

URBANA, IL 61801 



\section{PACIFIC JOURNAL OF MATHEMATICS EDITORS}

\author{
V. S. VARADARAJAN (Managing Editor) \\ University of California \\ Los Angeles, CA 90024 \\ Hebert Clemens \\ University of Utah \\ Salt Lake City, UT 84112 \\ Charles R. DePrima \\ California Institute of Technology \\ Pasadena, CA 91125
}

R. FINN

Stanford University

Stanford, CA 94305

HermanN FlaschKa

University of Arizona

Tucson, AZ 85721

RAMESH A. GANGOLlI

University of Washington

Seattle, WA 98195

ROBION KIRBY

University of California

Berkeley, CA 94720

ASSOCIATE EDITORS
R. ARENS
E. F. BECKENBACH
B. H. NEUMANN
F. WOLF
K. YoshidA (1906-1982)

C. C. Moore

University of California

Berkeley, CA 94720

H. SAMELSON

Stanford University

Stanford, CA 94305

HAROLD STARK

University of California, San Diego

La Jolla, CA 92093

\section{SUPPORTING INSTITUTIONS}

UNIVERSITY OF ARIZONA

UNIVERSITY OF BRITISH COLUMBIA

CALIFORNIA INSTITUTE OF TECHNOLOGY

UNIVERSITY OF CALIFORNIA

MONTANA STATE UNIVERSITY

UNIVERSITY OF NEVADA, RENO

NEW MEXICO STATE UNIVERSITY

OREGON STATE UNIVERSITY
UNIVERSITY OF OREGON

UNIVERSITY OF SOUTHERN CALIFORNIA

STANFORD UNIVERSITY

UNIVERSITY OF HAWAII

UNIVERSITY OF TOKYO

UNIVERSITY OF UTAH

WASHINGTON STATE UNIVERSITY

UNIVERSITY OF WASHINGTON 


\section{Pacific Journal of Mathematics}

Vol. 122, No. $1 \quad$ January, 1986

Michael James Cambern, Near isometries of Bochner $L^{1}$ and $L^{\infty}$ spaces ....1 Kun Soo Chang, Gerald William Johnson and David Lee Skoug, The

Feynman integral of quadratic potentials depending on two time

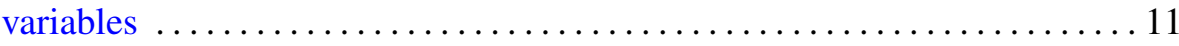

Robert Coleman, One-dimensional algebraic formal groups $\ldots \ldots \ldots \ldots \ldots 35$

Alberto Collino, The Abel-Jacobi isomorphism for the cubic fivefold .......43

N. J. Dev and S. S. Khare, Finite group action and vanishing of $N_{*}^{G}[F] \ldots 57$

Harold George Diamond and Jeffrey D. Vaaler, Estimates for partial sums

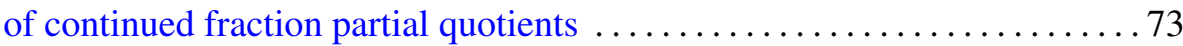

Kenneth R. Goodearl, Patch-continuity of normalized ranks of modules

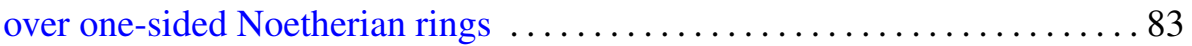

Dean Robert Hickerson and Sherman K. Stein, Abelian groups and

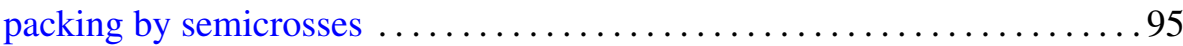

Karsten Johnsen and Harmut Laue, Fitting structures $\ldots \ldots \ldots \ldots \ldots 11$

Darren Long, Discs in compression bodies . ................... 129

Joseph B. Miles, On the growth of meromorphic functions with radially distributed zeros and poles ........................... 147

Walter Volodymyr Petryshyn, Solvability of various boundary value problems for the equation $x^{\prime \prime}=f\left(t, x, x^{\prime}, x^{\prime \prime}\right)-y \ldots \ldots \ldots \ldots \ldots . \ldots 169$

Elżbieta Pol, The Baire-category method in some compact extension problems

Masami Sakai, A new class of isocompact spaces and related results 211

Thomas Richard Shemanske, Representations of ternary quadratic forms and the class number of imaginary quadratic fields ..

Tsuyoshi Uehara, On class numbers of cyclic quartic fields 